\title{
Existence of Electrically Charged Structures with Regular Center in Nonlinear Electrodynamics Minimally Coupled to Gravity
}

\author{
Irina Dymnikova, ${ }^{1,2}$ Evgeny Galaktionov, ${ }^{1}$ and Eduard Tropp ${ }^{1}$ \\ ${ }^{1}$ A. F. Ioffe Physical-Technical Institute, Politekhnicheskaja 26, St. Petersburg, 194021, Russia \\ ${ }^{2}$ Department of Mathematics and Computer Science, University of Warmia and Mazury, Słoneczna 54, 10-710 Olsztyn, Poland \\ Correspondence should be addressed to Evgeny Galaktionov; evgalakt@mail.ru
}

Received 11 December 2014; Revised 12 February 2015; Accepted 9 March 2015

Academic Editor: Alkesh Punjabi

Copyright (c) 2015 Irina Dymnikova et al. This is an open access article distributed under the Creative Commons Attribution License, which permits unrestricted use, distribution, and reproduction in any medium, provided the original work is properly cited.

We address the question of correct description of Lagrange dynamics for regular electrically charged structures in nonlinear electrodynamics coupled to gravity. Regular spherically symmetric configuration satisfying the weak energy condition has obligatory de Sitter center in which the electric field vanishes while the energy density of electromagnetic vacuum achieves its maximal value. The Maxwell weak field limit $\mathscr{L}(F) \rightarrow F$ as $r \rightarrow \infty$ requires vanishing electric field at infinity. A field invariant $F$ evolves between two minus zero in the center and at infinity which makes a Lagrangian $\mathscr{L}(F)$ with nonequal asymptotic limits inevitably branching. We formulate the appropriate nonuniform variational problem including the proper boundary conditions and present the example of the spherically symmetric Lagrangian describing electrically charged structure with the regular center.

\section{Introduction}

The Abraham-Lorentz concept of a finite size electron which makes the total energy of the electron Coulomb field finite [1] encountered the problem of preventing the electron from flying apart under the Coulomb repulsion. Theories based on assumptions about the charge distribution were compelled to introduce cohesive forces of nonelectromagnetic origin (Poincaré stress), testifying that replacing a point charge with an extended one is impossible within the Maxwell electrodynamics since it demands introducing cohesive nonelectromagnetic forces [2].

Further search for the electron models was motivated by discovery of the Kerr-Newman solution to the EinsteinMaxwell equations [3]:

$$
\begin{aligned}
d s^{2}=- & d t^{2}+\frac{\Sigma}{\Delta} d r^{2}+\Sigma d \theta^{2}+\frac{\left(2 m r-e^{2}\right)}{\Sigma} \\
& \cdot\left(d t-a \sin ^{2} \theta d \phi\right)^{2}+\left(r^{2}+a^{2}\right) \sin ^{2} \theta d \phi^{2},
\end{aligned}
$$

$$
\begin{gathered}
A_{i}=-\frac{e r}{\Sigma}\left[1 ; 0,0,-a \sin ^{2} \theta\right], \\
\Sigma=r^{2}+a^{2} \cos ^{2} \theta, \quad \Delta=r^{2}-2 m r+a^{2}+e^{2},
\end{gathered}
$$

where $A_{i}$ is the associated electromagnetic potential.

Carter has found that the parameter a couples with the mass $m$ and charge $e$ independently, giving the angular momentum $J=m a$, an asymptotic magnetic momentum $\mu=e a$, and the same gyromagnetic ratio as predicted by the Dirac equation [4]. This suggested that the electron might be classically visualized as a massive charged source of the KerrNewman field [5].

Carter discovered also the nontrivial causality violation in the Kerr-Newman geometry in the case of a charged particle, when there are no Killing horizons, the manifold is geodesically complete (except for geodesics which reach the singularity), and any point can be connected to any other point by both a future and a past directed timelike curve. Closed timelike curves can extend over the whole space and cannot be removed by taking a covering space [4]. 
The Kerr-Newman solution belongs to the Kerr family of solutions to the source-free Einstein-Maxwell equations [4]. The source models for the Kerr-Newman exterior fields, involving a screening or covering causally dangerous region and Poincaré stress of different origins, can be roughly divided into disk-like [6-9], shell-like [5, 10-12], bag-like [13$20]$, and string-like ones ([21,22] and references therein). The problem of matching the Kerr-Newman exterior to a rotating material source does not have a unique solution, since one is free to choose arbitrarily the boundary between the exterior and the interior [6] as well as an interior model.

On the other hand, this question can be addressed in the frame of the model-independent mathematical approach [23], which was applied for introducing a quasilocal energy as dictated by employing a Hamilton-Jacobi analysis of the action functional rather than postulating quasilocal energy properties and then searching for a suitable expression for it [24].

In the spirit of this approach one can study equations describing a dynamical system to get information about its basic features. In the case of an electromagnetically interacting structure the appropriate equations come from nonlinear electrodynamics coupled to gravity (NED-GR).

NED theories appear as low-energy effective limits in certain models of string/M-theories [25-27]. Nonlinear electrodynamics was proposed by Born and Infeld in 1934 as founded on two basic principles: to consider electromagnetic field and particles within the frame of one physical entity which is electromagnetic field and to avoid letting physical quantities become infinite [28]. Electromagnetic energy was obtained finite by introducing an upper limit on the electric field related to the electron finite radius, but geometry remained singular [28].

Two basic points formulated by Born and Infeld can be realized in nonlinear electrodynamics coupled to gravity. Source-free NED-GR equations admit the class of regular causally safe axially symmetric asymptotically Kerr-Newman solutions which describe electromagnetic solitons representing spinning particles [29].

Theorems of nonexistence in NED-GR of electrically charged spherically symmetric structures with a regular center impose the condition of the Maxwell weak field limit in the center [30]. However, regular electrically charged structures can exist without the Maxwell center. The key point is that for any gauge-invariant Lagrangian $\mathscr{L}(F)$ stress-energy tensor of a spherically symmetric electromagnetic field has the algebraic structure such that

$$
T_{t}^{t}=T_{r}^{r}
$$

Regular spherically symmetric solutions with stress-energy tensors from the class (2) satisfying the weak energy condition (nonnegativity of density as measured by any local observer [31]) have obligatory de Sitter center [32-35]. In the NED-GR regular solutions [29, 36] interior de Sitter vacuum provides a needed cohesive force (an appearance of repulsive gravity was noted in the early electron models ([37] and references therein)) and proper cut-off on the field selfinteraction divergent for a point charge $[36,38]$.
The regular spherical solutions generated by (2) belong to the Kerr-Schild class $[19,39,40]$ and can be transformed by the Gürses-Gürsey algorithm [41] into regular axially symmetric asymptotically Kerr-Newman solutions describing electrically charged black holes and solitons. De Sitter center transforms into the interior de Sitter disk which behaves like perfect conductor and ideal diamagnetic and displays superconducting behaviour within a single spinning charged structure [29]. The mass of an object is the finite positive electromagnetic mass generically related to interior de Sitter vacuum and breaking of space-time symmetry from the de Sitter group in the origin [29, 34, 36, 42, 43].

The price for such a promising road is unavoidable branching of a spherically symmetric Lagrangian for regular electrically charged NED-GR configurations.

The generic feature of any regular electromagnetic spherically symmetric structure satisfying the weak energy condition is the de Sitter center where Lagrangian takes the value $\mathscr{L}=2 \rho(0)$ as $r \rightarrow 0$ [36]. Regularity requires $F \rightarrow-0$ as $r \rightarrow 0[30,36]$. In the limit $r \rightarrow \infty$ Lagrangian follows the Maxwell weak field limit, $\mathscr{L}(F) \rightarrow F \rightarrow$ -0 . Electromagnetic invariant $F$ evolves thus between minus zeros at the center and at infinity with at least one minimum in between. Nonmonotonic behaviour of the invariant $F$ leads unavoidably to branching of any spherically symmetric gauge-invariant Lagrangian $\mathscr{L}(F)[30,36]$.

Regular spherically symmetric solutions with the nonzero electric charge [36, 44-47] were found with using the alternative $P$-form of nonlinear electrodynamics obtained from the standard Lagrangian $F$-form by the Legendre transformation [48]. F-P duality turns into electric-magnetic duality in the Maxwell limit but in general case it connects different theories [30] which is clearly manifested by branching of a Lagrangian in the $F$ frame.

The aim of this paper is to formulate the correct variational problem of the Lagrange dynamics for the regular electrically charged spherically symmetric NED-GR configurations.

The paper is organized as follows. In Section 2 we present the basic equations and in Section 3 we outline the generic features of regular electrically charged configurations. Section 4 is devoted to the variational problem of the Lagrange dynamics. In Section 5 we present the particular Lagrangian responsible for the regular electrically charged solution [36]. In Section 6 we summarize and discuss the results. Appendix contains the detailed consideration concerning definition of the stress-energy tensor for an electromagnetic field.

\section{Basic Equations}

In nonlinear electrodynamics minimally coupled to gravity, the action is given (in geometrical units $c=G=1$ ) by

$$
S=\frac{1}{16 \pi} \int d^{4} x \sqrt{-g}[R-\mathscr{L}(F)] ; \quad F=F_{\mu \nu} F^{\mu \nu} .
$$

Here $R$ is the scalar curvature, and $F_{\mu \nu}=\partial_{\mu} A_{\nu}-\partial_{\nu} A_{\mu}$ is the electromagnetic field. The gauge-invariant electromagnetic 
Lagrangian $\mathscr{L}(F)$ is an arbitrary function of $F$ which should have the Maxwell limit, $\mathscr{L} \rightarrow F, \mathscr{L}_{F}=d \mathscr{L} / d F \rightarrow 1$ in the weak field regime.

Action (3) gives the dynamic field equations

$$
\nabla_{\mu}\left(\mathscr{L}_{F} F^{\mu \nu}\right)=0
$$

The Bianchi identities yield

$$
\nabla_{\mu}^{*} F^{\mu \nu}=0
$$

An asterisk denotes the Hodge dual [49]; the antisymmetric unit tensor is chosen in such a way that $\eta_{0123}=\sqrt{-g}$.

The Einstein equations can be written in the form [30]

$$
\begin{aligned}
G_{v}^{\mu} & =-T_{\nu}^{\mu}=2 \mathscr{L}_{F} F_{\nu \beta} F^{\mu \beta}-\frac{1}{2} \delta_{\nu}^{\mu} \mathscr{L} \\
& =\frac{1}{2} \operatorname{diag}\left(\mathscr{L}-2 F \mathscr{L}_{F}, \mathscr{L}-2 F \mathscr{L}_{F}, \mathscr{L}, \mathscr{L}\right) .
\end{aligned}
$$

The definition of $T_{\mu \nu}$ in (6) differs from the standard definition (see, e.g., $[49,50]$ ) by $8 \pi$, so that $T_{t(\text { here })}^{t}=8 \pi \rho$ and so forth.

The density and pressures for electrically charged structures are given by

$$
\rho=-p_{r}=\left[\frac{1}{2} \mathscr{L}-F \mathscr{L}_{F}\right] ; \quad p_{\perp}=-\frac{1}{2} \mathscr{L} .
$$

It follows from (7) that a spherically symmetric electromagnetic field with an arbitrary gauge-invariant Lagrangian $\mathscr{L}(F)$ has stress-energy tensor with the algebraic structure (2).

Symmetry of source term (2) leads to the metric

$$
d s^{2}=f(r) d t^{2}-\frac{d r^{2}}{f(r)}-r^{2} d \Omega^{2},
$$

where $d \Omega^{2}$ is the line element on a unit sphere. The metric function and mass function are given by

$$
f(r)=1-\frac{2 \mathscr{M}(r)}{r} ; \quad \mathscr{M}(r)=\frac{1}{2} \int_{0}^{r} \rho(x) x^{2} d x .
$$

Radial pressure satisfies, in accordance with (2), $p_{r}=-\rho$, and tangential pressure is given by

$$
p_{\perp}=-\rho-\frac{r}{2} \rho^{\prime}
$$

Metric (9) has the de Sitter asymptotic for $r \rightarrow 0$ which directly follows from (2)

$$
f(r)=1-\frac{\Lambda}{3} r^{2} ; \quad \Lambda=\rho(0) ; \quad p_{\perp}=-\rho
$$

and the Reissner-Nordström asymptotic for $r \rightarrow \infty$ dictated by the Maxwell limit

$$
f(r)=1-\frac{2 m}{r}+\frac{q^{2}}{r^{2}}
$$

In the spherically symmetric case the only essential component of $F_{\mu \nu}$ describing a radial electric field is $F_{01}=-E(r)$. Dynamical equations (4) yield [30]

$$
r^{2} \mathscr{L}_{F} F^{01}=q,
$$

where $q$ is constant of integration identified as an electric charge by asymptotic behavior in the weak field limit. The field invariant $F$ is given by

$$
F=2 F_{01} F^{01}=-\frac{2 q^{2}}{\mathscr{L}_{F}^{2} r^{4}} .
$$

Equation (7) implies

$$
p_{\perp}+\rho=-F \mathscr{L}_{F} .
$$

The weak energy condition requires $\rho \geq 0$ and $p_{\perp}+\rho \geq 0$ [31] which imposes two general constraints on the Lagrangian $\mathscr{L}(F)$. Equations (7) and (15) lead to

$$
\begin{gathered}
\mathscr{L}_{F} \geq 0, \\
\mathscr{L}(F) \geq 2 F \mathscr{L}_{F} .
\end{gathered}
$$

\section{Generic Features of Field Dynamics}

The theorem of nonexistence in NED-GR of electrically charged configurations with the regular center requires the Maxwell behavior at the regular center, $\mathscr{L} \rightarrow F, \mathscr{L}_{F} \rightarrow 1$ as $F \rightarrow 0$ [30]. The proof is that regularity of stress-energy tensor requires $\left|F \mathscr{L}_{F}\right|<\infty$ as $r \rightarrow 0$, while $F \mathscr{L}_{F}^{2} \rightarrow-\infty$ by virtue of (14); it follows that $\mathscr{L}_{F} \rightarrow \infty$, while $F \rightarrow 0$, which is strongly non-Maxwell behavior.

Strictly speaking, this theorem tells us that a regular electrically charged structure cannot exist with the Maxwell weak field limit $\mathscr{L} \rightarrow F$ as $r \rightarrow 0$.

However, a regular electromagnetic structure can exist without the Maxwell limit in the centre [36]. Regularity of a stress-energy tensor of an electromagnetic field requires $T_{t}^{t}=\rho<\infty$. Algebraic structure of a stress-energy tensor (2) leads to the existence of de Sitter center in regular solutions [34], where electromagnetic density $\rho$ achieves its maximum, since the weak energy condition requires, by virtue of (10), $\rho^{\prime} \leq 0$, and one cannot expect validity of the weak field limit in the region of the maximal density. As a result one obtains a regular electrically charged spherically symmetric structure with the de Sitter center obligatory for all solutions of class (2), in which field tension must go to zero (by requirement of regularity), while the energy density of the electromagnetic vacuum $T_{t}^{t}$ achieves its maximal finite value which represents the de Sitter cut-off on the self-energy density [36].

Requirement of regularity demands inevitably $F \rightarrow-0$ and $F \mathscr{L}_{F}^{2} \rightarrow-\infty[30,36]$. De Sitter asymptotic implies $p_{\perp}+$ $\rho=0$ which leads to $F \mathscr{L}_{F}=0$ at $r=0$. It follows, with taking into account (16) and (14), $\mathscr{L}_{F} \rightarrow+\infty$ when $r \rightarrow 0$. Conditions for a Lagrangian in the regular center where $F \rightarrow$ -0 are thus

$$
\begin{gathered}
\mathscr{L}(0)=2 \rho(0), \\
\mathscr{L}_{F} \longrightarrow \infty \text { as } r \longrightarrow 0 .
\end{gathered}
$$




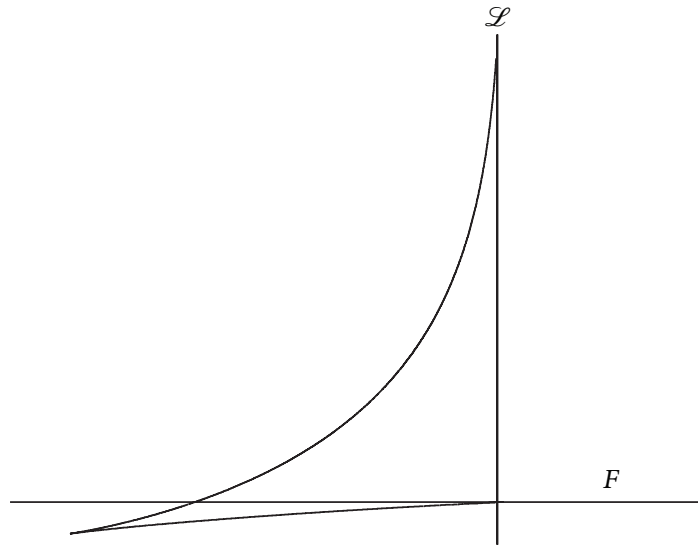

Figure 1: Typical behavior of a Lagrangian. Cusp corresponds to $F^{\prime}=0$.

The Maxwell asymptotic at $r \rightarrow \infty$ imposes two conditions on Lagrangian in the limit $F \rightarrow-0$ :

$$
\begin{gathered}
\mathscr{L} \longrightarrow F \longrightarrow-0, \\
\mathscr{L}_{F} \longrightarrow 1 .
\end{gathered}
$$

Regularity requires $F \rightarrow-0$ as $r \rightarrow 0$, while the Maxwell limit requires $F \rightarrow-0$ as $r \rightarrow \infty$. The invariant $F$ is not a monotonic function of $r$ evolving between two minus zeros and having at least one minimum in between. It leads unavoidably to branching of a Lagrangian [30, 36]. Lagrangian on its way from (18) to (19) must change its sign. Additional branching would require additional change of the sign. According to (7), the sign of a Lagrangian is opposite to the sign of tangential pressure which can vanish only once for the case of one de Sitter vacuum scale [34] (for the case of several vacuum scales see [51]); so in the considered case we deal with two branches of a Lagrangian. Equation (14) defines in the first approximation the derivative $d \mathscr{L}_{F} / d F$ in the minimum $r=r_{c}$ of the invariant $F$ by

$$
\mathscr{L}_{F F}=-\frac{2 \mathscr{L}_{F}}{\left(F^{\prime} r_{c}\right)} .
$$

In accordance with (16), $\mathscr{L}_{F}$ has the same finite limit as $F \rightarrow$ $F_{c}+0$ and $F \rightarrow F_{c}-0$, while $F^{\prime}$ changes its sign in the minimum, so that $\mathscr{L}_{F F}$ tends to infinities of opposite signs. Therefore a Lagrangian $\mathscr{L}(F)$ has the cusp at $F=F_{c}$.

The Lagrangian $\mathscr{L}(F)$ is a monotonic function of $F$; when the weak energy condition is satisfied, $\mathscr{L}_{F} \geq 0$ according to (16). A monotonic function $\mathscr{L}(F)$ first decreases smoothly along the first branch from its maximal value $\mathscr{L}(0)=2 \rho(0)$ to its value in the cusp $\mathscr{L}_{c}$ as $F$ decreases from $F=-0$ at $r=0$ to $F_{\min }=F_{c}$; then the Lagrangian increases along the second branch from its minimal value $\mathscr{L}_{c}<0$ to its Maxwell limit $\mathscr{L} \rightarrow F \rightarrow-0$ as $F$ increases from $F_{c}$ to $F \rightarrow-0$ as $r \rightarrow \infty$. Typical behavior of the Lagrangian as a function of $F$ is shown in Figure 1.

On the other hand, a dynamical system represents a physical object existing in the space within the range $0 \leq r \leq \infty$.
So we can present a Lagrangian in the parametric form combining $\mathscr{L}(F)$ with $F(r)$. Behaviour of Lagrangian as a function of $r$, according to (7), follows the behavior of tangential pressure up to the opposite sign, $\mathscr{L}=-2 p_{\perp}$. The Lagrangian $r$-derivative $\mathscr{L}^{\prime}=\mathscr{L}_{F} F^{\prime}$ is finite everywhere and vanishes in the minimum of the invariant $F(r)$. Value $\mathscr{L}_{F}$ is finite and nonzero there by (15), since $p_{\perp}+\rho$ vanishes only at the center and infinity.

Second derivative is given by

$$
\frac{d^{2} \mathscr{L}}{d r^{2}}=\frac{d \mathscr{L}_{F}}{d r} F^{\prime}+\mathscr{L}_{F} F^{\prime \prime}
$$

It is finite and positive in the extremum at $r=r_{c}$; hence Lagrangian achieves its minimum in the minimum of the invariant $F$. At the same time it is the maximum of the tangential pressure. We find that the invariant $F$ and Lagrangian $\mathscr{L}(r)$ achieve their minimum at the surface $r=r_{c}$ where $p_{\perp}(r)$ has its maximum.

As a function of $r$, Lagrangian does not demonstrate a branching. Its $r$-derivative is negative in the region $0 \leq$ $r<r_{c}$ and positive in the region $r_{c}<r<\infty$. Starting from its maximal value $\mathscr{L}(0)$, the Lagrangian decreases to its minimum $\mathscr{L}_{c}$ and then increases to $\mathscr{L}=-0$ as $r \rightarrow \infty$.

Parametric presentation of a Lagrangian is admissible but not enough for studying field dynamics described by the dependence $\mathscr{L}(F)$. Branching of Lagrangian as a function of $F$ enforces introduction of two Lagrangians. In terms of the variational calculus we deal with nonuniform variational problem typically applied for the case of two media. In the next section we apply this approach for actually one medium, nonlinear electromagnetic field, divided in two parts by nonmonotonic behaviour of the field invariant $F$ and derive proper conditions on their boundary $r=r_{c}$ where $F$ has the minimum.

\section{Variational Problem}

Nonuniform variational problem in our case is presented by the action

$$
\begin{aligned}
& S=S_{\text {int }}+S_{\text {ext }} \\
&=\frac{1}{16 \pi}\left[\int_{\Omega_{\text {int }}}\left(R-\mathscr{L}_{\text {int }}(F)\right) \sqrt{-g} d^{4} x\right. \\
& \\
&\left.\quad+\int_{\Omega_{\text {ext }}}\left(R-\mathscr{L}_{\text {ext }}(F)\right) \sqrt{-g} d^{4} x\right] .
\end{aligned}
$$

Asymptotic behavior of the electromagnetic Lagrangians is given by

$$
\begin{gathered}
\mathscr{L}_{\mathrm{int}}(F) \longrightarrow 2 \rho_{0} ; \quad \mathscr{L}_{F(\mathrm{int})} \longrightarrow+\infty \\
\text { as } F \longrightarrow-0 \quad \text { at } r \longrightarrow 0, \\
\mathscr{L}_{\mathrm{ext}}(F) \longrightarrow F ; \quad \mathscr{L}_{F(\mathrm{ext})} \longrightarrow 1 \\
\text { as } F \longrightarrow-0 \quad \text { at } r \longrightarrow \infty .
\end{gathered}
$$

Each part of the action includes the Hilbert action $S_{G}$ and the matter (electromagnetic) action $S_{M}$. In the case of a minimal coupling, $S_{G}$ does not depend on a matter field. Variation 
of the total action with respect to electromagnetic variables yields the same dynamical field equations as variation of $S_{M}$ only.

Each part of the manifold, $\Omega_{\text {int }}$ and $\Omega_{\text {ext }}$ is confined by the initial and final spacelike hypersurfaces $t_{\text {in }}$ and $t_{\text {fin }}$, by the timelike 3-surface $\Sigma_{3}$ extended to infinity where electromagnetic field vanishes and by the timelike internal common boundary between $\Omega_{\text {int }}$ and $\Omega_{\text {ext }}$ denoted as $\Sigma_{c}$. This surface is not arbitrary but distinguished by the extremum of the field invariant $F$. Our task is to find the proper conditions on the internal boundary surface $\Sigma_{c}$ to guarantee satisfying the dynamical and the Einstein equations.

Dynamical variables in the Lagrangian $\mathscr{L}(F)$ are electromagnetic potentials $A_{\mu}$. At the surfaces $t_{\text {in }}$ and $t_{\text {fin }}$ their variations should satisfy $\delta A_{\mu}=0$.

Electromagnetic tensor $F_{\mu \nu}=\partial_{\nu} A_{\mu}-\partial_{\mu} A_{\nu}=-F_{\nu \mu}$ satisfies $F_{\mu \nu} \delta F^{\mu \nu}=F^{\mu \nu} \delta F_{\mu \nu}$. Variation of $S_{M}$ reduces to

$$
\begin{aligned}
\delta S_{M}= & \frac{1}{4 \pi} \int_{\Omega_{\text {int }}} \mathscr{L}_{F(\text { int })} F_{\text {int }}^{\mu \nu} \frac{\partial}{\partial x^{\nu}} \delta A_{\mu(\text { int })} \sqrt{-g} d \Omega \\
& +\frac{1}{4 \pi} \int_{\Omega_{\text {ext }}} \mathscr{L}_{F(\text { ext })} F_{\text {ext }}^{\mu \nu} \frac{\partial}{\partial x^{\nu}} \delta A_{\mu(\text { ext })} \sqrt{-g} d \Omega .
\end{aligned}
$$

Integrating by parts with using the Gauss theorem we get

$$
\begin{aligned}
\delta S_{M}= & \frac{1}{4 \pi} \int_{\Sigma_{\text {int }}} \mathscr{L}_{F(\text { int })} F_{\text {int }}^{\mu \nu} \delta A_{\mu(\text { int })} \sqrt{-g} d \sigma \\
& +\frac{1}{4 \pi} \int_{\Sigma_{\text {ext }}} \mathscr{L}_{F(\text { ext })} F_{\text {ext }}^{\mu \nu} \delta A_{\mu(\text { ext })} \sqrt{-g} d \sigma \\
& +\frac{1}{4 \pi} \int_{\Omega_{\text {int }}}\left(\mathscr{L}_{F(\text { int })} \nabla_{\nu} F_{\text {int }}^{\mu \nu}\right) \delta A_{\mu(\text { int })} \sqrt{-g} d \Omega \\
& +\frac{1}{4 \pi} \int_{\Omega_{\text {ext }}}\left(\mathscr{L}_{F(\text { ext })} \nabla_{\nu} F_{\text {ext }}^{\mu \nu}\right) \delta A_{\mu(\text { ext })} \sqrt{-g} d \Omega .
\end{aligned}
$$

$\delta S_{M}=0$ requires fulfilment of the dynamical Euler equations $\nabla_{\nu} F^{\mu \nu}=0$ in both parts of the manifold and vanishing surface terms. The choice of the external boundaries $t_{\mathrm{in}}, t_{\mathrm{fin}}$, and $\Sigma_{3}$ guarantees vanishing of surface contributions. On the internal boundary $\Sigma_{c}$ we should have

$$
\int_{\Sigma_{c}}\left[\mathscr{L}_{F(\text { int })} F_{\text {int }}^{\mu \nu}\left(\delta A_{\mu}\right)_{\text {int }}-\mathscr{L}_{F(\text { ext })} F_{\text {ext }}^{\mu \nu}\left(\delta A_{\mu}\right)_{\text {ext }}\right] \sqrt{-g} d \sigma_{\nu}=0 .
$$

The surface $\Sigma_{c}$ is specified by the condition of continuity of the dynamical variables

$$
A_{\mathrm{int}}^{\mu}=A_{\mathrm{ext}}^{\mu} .
$$

As a result we get

$$
\int_{\Sigma_{c}}\left(\mathscr{L}_{F(\mathrm{int})} F_{\mu \nu(\mathrm{int})}-\mathscr{L}_{F(\mathrm{ext})} F_{\mu \nu(\mathrm{ext})}\right) \sqrt{-g} \delta A^{\mu} d \sigma^{\nu}=0 .
$$

In the spherically symmetric static case the only nonzero field component $F_{01}=-\partial A_{0} / \partial r$. Introducing $A^{0}=\phi$ and $F_{01}=-\partial \phi / \partial r=-E(r)$, we write $(28)$ in the explicit form $\phi_{\text {int }}=\phi_{\text {ext }}$, and taking into account that variation $\delta \phi$ is arbitrary, we obtain the condition imposed on the electromagnetic variables on the boundary $\Sigma_{c}$ :

$$
\mathscr{L}_{F(\text { int })} E_{\text {int }}=\mathscr{L}_{F(\text { ext })} E_{\text {ext }} .
$$

For spherically symmetric nonlinear electromagnetic field dielectric permeability $\epsilon=\mathscr{L}_{F}$ [48], condition (30) requires thus continuity of the electric induction $D=\epsilon E$ on $\Sigma_{c}$ :

$$
D_{\text {int }}=D_{\text {ext }} \text {. }
$$

Variation of the total action $S=S_{G}+S_{M}$ in each part of the manifold with respect to $g_{\mu \nu}$ yields the Einstein equations $G_{\mu \nu}=R_{\mu \nu}-(1 / 2) R g_{\mu \nu}=-T_{\mu \nu}$, where

$$
T_{\mu \nu}=\frac{4 \pi}{\sqrt{-g}} \frac{\delta S_{M}}{\delta g^{\mu \nu}}
$$

is the stress-energy tensor. For a diffeomorphism invariant action, the conservation equation $\nabla^{\mu} T_{\mu \nu}=0$ is fulfilled by virtue of the dynamic field equations (detailed consideration in Appendix). The conservation equation $\nabla_{\mu} T^{\mu \nu}=0$ imposes additional conditions on the boundary surface $\Sigma_{c}$. Vanishing a surface term under variation with respect to $g_{\mu \nu}$ requires $T_{\mu \nu}$ to satisfy on the boundary $\Sigma_{c}$

$$
\int_{\Sigma_{c}}\left[T_{\nu(\text { int })}^{\mu}-T_{\nu(\text { ext })}^{\mu}\right] \sqrt{-g} w^{\nu} d \sigma_{\mu}=0,
$$

where $w^{\nu}$ is an arbitrary vector field. In our case $\Sigma_{c}$ is the sphere, $T_{\nu}^{\mu}$ satisfies (2) and (7), and condition (33) reduces to

$$
\int_{\Sigma_{c}}\left[T_{1(\text { int })}^{1}-T_{1(\text { ext })}^{1}\right] w^{1} \sqrt{-g} d \sigma_{1}=0
$$

which results, due to arbitrariness of $w^{\mu}$, in

$$
\mathscr{L}_{\text {(int) }}-2 \mathscr{L}_{F(\text { int })} F_{\text {int }}=\mathscr{L}_{\text {(ext) }}-2 \mathscr{L}_{F(\text { ext })} F_{\text {ext }} .
$$

Condition (35) requires, by virtue of (7), continuity of density on the surface $\Sigma_{c}$ :

$$
\rho_{\text {int }}=\rho_{\text {ext }} \cdot
$$

Conditions (30) and (35) are the Erdmann-Weierstrass conditions obtained here for the Lagrange density $\mathscr{L}(F)$. (In the textbooks on variational calculus they are typically derived for a function $\Phi\left(x, y_{i}, y_{i}^{\prime}\right)$ (corresponding to a Lagrange function $L$ ) for the cases of discontinuity of derivatives of dynamical variables $y_{i}$.) In the case of continuous invariant $F$, considered here, conditions (30) and (35) are satisfied automatically and lead, with taking into account generic behavior of a Lagrangian, $\mathscr{L}_{\text {int }}=\mathscr{L}_{\text {ext }}$ on $\Sigma_{c}$, to $E_{\text {ext }}=$ $E_{\text {int }}, \mathscr{L}_{F(\text { int })}=\mathscr{L}_{F(\text { ext })}$, and $p_{\perp(\text { int })}=p_{\perp(\text { ext })}$ on $\Sigma_{c}$. For a discontinuous invariant $F$ the situation will be different [52].

Now let us consider conditions arising on $\Sigma_{c}$ under variation with respect to $g_{\mu \nu}$. The boundary terms coming from the integration by parts and applying the Gauss theorem have the form $[49,50]$

$$
\int_{\Omega} g^{\mu v} \delta R_{\mu \nu} \sqrt{-g} d^{4} x=\int_{\Sigma} \sqrt{-g} v^{\mu} d \sigma_{\mu}
$$


where

$$
v^{\nu}=g^{\alpha \beta} \delta \Gamma_{\alpha \beta}^{\nu}-g^{\nu \mu} \delta \Gamma_{\mu \beta}^{\beta} .
$$

Each boundary term in (37) vanishes if both metric tensor $g^{\mu \nu}$ and its first derivatives are held fixed on the boundary; that is, vanishing of boundary contributions requires vanishing $\delta \Gamma_{\mu \nu}^{\beta}$ on the surface $\Sigma$ [49]. Less restrictive and more informative conditions on the metric tensor at the boundary $\Sigma$ require only $\delta g_{\mu \nu}=0$. The first integral in (37) can be written as [50]

$$
\int_{\Omega} g^{\mu \nu} \delta R_{\mu \nu} \sqrt{-g} d^{4} x=\int_{\Sigma} v_{\mu} n^{\mu},
$$

where $n^{\mu}$ is the unit normal to the boundary $\Sigma$. With using (38) we have on $\Sigma[50]$

$$
\begin{gathered}
v_{\alpha} n^{\alpha}=n^{\alpha} h^{\mu \nu}\left[\nabla_{\nu}\left(\delta g_{\alpha \mu}\right)-\nabla_{\alpha}\left(\delta g_{\mu \nu}\right)\right], \\
h_{\mu \nu}=g_{\mu \nu} \pm n_{\mu} n_{\nu}
\end{gathered}
$$

where $h_{\mu \nu}$ is the induced metric on $\Sigma$. The first term in (40) vanishes by virtue of $\delta g_{\mu \nu}=0$ on $\Sigma$. The second term is related to the trace of the extrinsic curvature of the boundary $K=K_{\alpha}^{\alpha}=h_{\beta}^{\alpha} \nabla_{\alpha} n^{\beta}$, its variation $2 \delta K=n^{\alpha} h^{\mu \nu} \nabla_{\alpha}\left(\delta g_{\mu \nu}\right)$ [50]. It follows the appearance of the additional term in the action

$$
\delta S_{G}=\int_{\Omega} G_{\alpha \beta} \delta g^{\alpha \beta} \sqrt{-g} d^{4} x-2 \int_{\Sigma} \delta K
$$

and the unwanted contribution can be excluded by modifying $S_{G}$ to $S_{G}+2 \int_{\Sigma} K[50]$.

On the internal boundary surface $\Sigma_{c}$ we have two contributions from (40). The first of them vanishes since the metric function (9) is regular (the surface $r=r_{c}$ is not distinguished in the metric), and continuity of the function $\rho(x)$ in the integral in (9) is guaranteed by condition (36). The second term in (40) vanishes since the condition for the extrinsic curvature $K_{\text {int }}=K_{\text {ext }}$ on $\Sigma_{c}$ is satisfied in this case.

\section{Example}

The regular spherically symmetric solution with the regular center presented in [36] was obtained in the $P$-frame of NED-GR with the Hamiltonian-like function $\mathscr{H}(P)=P(1+$ $\alpha \sqrt{-P})^{2}$, where $P=-2 q^{2} / r^{4}$, which gives the regularized Coulomb profile [36]

$$
\rho(r)=\frac{q^{2}}{\left(r^{2}+r_{q}^{2}\right)^{2}} .
$$

Its value in the center gives the proper expression for a cut-off on self-energy density.

The metric function [36]

$$
f(r)=1-\frac{4 m}{\pi r}\left(\operatorname{arctg} \frac{r}{r_{q}}-\frac{r r_{q}}{r^{2}+r_{q}^{2}}\right)
$$

has the de Sitter asymptotic $f(r)=1-r^{2} / r_{0}^{2}$ and $r_{0}^{2}=3 / \rho(0)$ as $r \rightarrow 0$ and the Reissner-Nordström asymptotic (12) as $r \rightarrow \infty$.

The characteristic parameter $\eta=2 r_{g} /\left(\pi r_{q}\right)$, where $r_{g}=2 m$ is the Schwarzschild radius, defines the type of a spherically symmetric structure with the regular de Sitter center: a regular electrically charged black hole for $\eta \geq \eta_{\text {crit }}=$ 2.816 with at most two horizons, the double horizon $r_{ \pm}=$ $1.825 r_{q}$ for $\eta=\eta_{\text {crit }}$, and electrically charged soliton for $\eta<\eta_{\text {crit }}[36]$.

The invariant $F(r)$ and Lagrangian $\mathscr{L}(r)$ are given by [36]

$$
F(r)=-\frac{2 q^{2} r^{8}}{\left(r^{2}+r_{q}^{2}\right)^{6}} ; \quad \mathscr{L}(r)=\frac{2 q^{2}\left(r_{q}^{2}-r^{2}\right)}{\left(r^{2}+r_{q}^{2}\right)^{3}} .
$$

The minimum of $F$ is achieved at $r_{c}=\sqrt{2} r_{q}$. Normalizing $r$ on $r_{q}$ and $F$ on $q^{2} / r_{q}^{4}$, we write equations in the dimensionless form and resolve them with respect to $z=\left(r / r_{q}\right)^{2}$ to obtain $\mathscr{L}_{\text {int }}$ and $\mathscr{L}_{\text {ext }}$ as the functions $F$.

We find that the regular solution presented in [36] can be obtained in the $F$ frame of the Lagrange dynamics with the Lagrangians

$$
\begin{gathered}
\mathscr{L}_{\text {int }}(F)=\frac{2\left(1-z_{2}(\beta)\right)}{\left(z_{2}(\beta)+1\right)^{3}}, \\
z_{2}=-\frac{2}{3} \frac{\sqrt{1-6 \beta}}{\beta} \cos \left(\frac{\alpha}{3}+\frac{\pi}{3}\right)-1+\frac{1}{3 \beta}, \\
\mathscr{L}_{\text {ext }}(F)=\frac{2\left(1-z_{1}(\beta)\right)}{\left(z_{1}(\beta)+1\right)^{3}}, \\
z_{1}=\frac{2}{3} \frac{\sqrt{1-6 \beta}}{\beta} \cos \frac{\alpha}{3}-1+\frac{1}{3 \beta}, \\
\beta=\sqrt{-\frac{F}{2}}, \quad \beta \in\left(0, \frac{4}{27}\right) \\
\alpha=\arccos \left(\frac{2-18 \beta+27 \beta^{2}}{2(1-6 \beta)^{3 / 2}}\right) .
\end{gathered}
$$

For $\mathscr{L}_{\text {int }}$ parameter $z_{2}$ changes within the range $0 \leq z_{2} \leq 2$ and for $\mathscr{L}_{\text {ext }}$ we have $2 \leq z_{1}<\infty$. Minimal value of the invariant $F$ is $F_{\min }=-2^{5} \cdot 3^{-6}$. In the minimum of the invariant $\mathscr{L}_{\text {int }}=\mathscr{L}_{\text {ext }}=-2 / 27$, and $d \mathscr{L}_{\text {int }} / d F=d \mathscr{L}_{\text {ext }} / d F=$ 3.395. Second derivatives go to infinities of the opposite signs: $d^{2} \mathscr{L}_{\text {ext }} / d F^{2} \rightarrow-\infty$, while $d^{2} \mathscr{L}_{\text {int }} \rightarrow+\infty$.

For $F \rightarrow-0$, we have $\beta \rightarrow 0$, and the parameter $\alpha \rightarrow 0$. Then $z_{2} \rightarrow 0$ and $\mathscr{L}_{\text {int }} \rightarrow 2$, while $\mathscr{L}_{\text {ext }} \rightarrow$ $F \rightarrow-0$. The Lagrangian $\mathscr{L}_{\text {int }}$ evolves from $\mathscr{L}_{\text {int }}(0)=2$ to $\mathscr{L}_{\text {int }}\left(F_{\text {min }}\right)=-2 / 27$. The external Lagrangian starts from $\mathscr{L}_{\text {ext }}\left(F_{\text {min }}\right)=-2 / 27$ and evolves to $\mathscr{L}_{\text {ext }}(r \rightarrow \infty)=-0$.

\section{Summary and Discussion}

We have proved the existence of spherically symmetric electrically charged structures with the regular center in nonlinear electrodynamics coupled to gravity. They are described 
in the frame of the Lagrange dynamics by the nonuniform variational problem with the action

$$
\begin{aligned}
S=\frac{1}{16 \pi}\left[\int_{\Omega_{\mathrm{int}}}\left(R-\mathscr{L}_{\mathrm{int}}(F)\right) \sqrt{-g} d^{4} x\right. \\
\left.\quad+\int_{\Omega_{\mathrm{ext}}}\left(R-\mathscr{L}_{\mathrm{ext}}(F)\right) \sqrt{-g} d^{4} x\right] .
\end{aligned}
$$

Introduction of two Lagrangians is enforced by behaviour of the field invariant $F$ which must vanish at $r=0$ to guarantee regularity and at infinity to satisfy the Maxwell weak field limit.

In the case of continuous invariant $F$ the nonuniform variational problem (46) describes nonlinear electromagnetic field as a medium with amazingly uniform properties: equality not only of electric induction but also of electric field tension on the internal boundary surface $\Sigma_{c}$ and in consequence equality of the dielectric permeability. The situation can be essentially different for a rotating electrically charged regular structure.

Introduction of two Lagrangians for one electromagnetic medium suggests nontrivial field dynamics. It could, for example, involve a phase transition related to interior de Sitter vacuum $[53,54]$. In the case of a charged regular black hole or soliton a phase transition can be related to dual electrodynamics [19]. A particular example is regular structure of a hybrid type [55] in which an electrically charged exterior region is matched to a magnetically charged core.

Regular spherically symmetric solutions of the Einstein equations satisfying the weak energy condition and specified by $T_{t}^{t}=T_{r}^{r}$ have obligatory de Sitter center $[32,34]$, belong to the Kerr-Schild class [40], and can be transformed into regular spinning solutions by the Gürses-Gürsey algorithm [41] which transforms the de Sitter center to the de Sitter vacuum disk. It has properties of a perfect conductor and ideal diamagnetic and displays superconducting behavior within a single spinning object [29].

Interior de Sitter vacuum supplies a structure with the finite positive electromagnetic mass related to breaking of space-time symmetry from the de Sitter group in the origin, which can explain existence of negative mass-squared differences for neutrino and predict a $\mathrm{TeV}$ scale for gravitoelectroweak unification [42].

Many modern theories propose two key modifications on the way to unify gravity with standard model of particle physics: the existence of additional space dimensions and the existence of a minimal length scale related to maximal resolution.

Existence of minimal length was observed in the annihilation reaction $e^{+} e^{-} \rightarrow \gamma \gamma(\gamma)$ which puts the limit on maximal resolution at the scale $E=1.253 \mathrm{TeV}$ with the $5 \sigma$ reliability. Related minimal length $l_{e}=1.57 \times 10^{-17} \mathrm{~cm}$ appears naturally in the NED-GR context as a distance of the closest approach of annihilating particles at which the electromagnetic attraction is balanced by the gravitational repulsion of the interior de Sitter vacuum [38].

\section{Appendix}

\section{Stress-Energy Tensor}

The matter action $S_{M}(\psi)$ should be invariant under diffeomorphisms [50] which gives

$$
\int \frac{\delta S_{M}}{\delta g^{\mu \nu}} \delta g^{\mu \nu}+\int \frac{\delta S_{M}}{\delta \psi} \delta \psi=0
$$

with

$$
\delta g^{\mu \nu}=2 \nabla^{(\mu} w^{\nu)}
$$

where $w^{\mu}$ is an arbitrary vector field. When matter field $\psi$ satisfies the dynamical field equations, then $\delta S_{M} / \delta \psi=0$, and this leads, with taking into account (32), to [49]

$$
\begin{aligned}
& \int \sqrt{-g} T_{\mu \nu} \nabla^{(\mu} w^{\nu)} d^{4} x \\
& =0=-\int \nabla^{\mu} T_{\mu \nu} w^{\nu} \sqrt{-g} d^{4} x+\int_{\Sigma} \sqrt{-g} T_{\mu \nu} w^{\nu} d \sigma^{\mu}
\end{aligned}
$$

The second term on the right-hand side vanishes for all smooth $w^{\mu}$ of compact support which results in $\nabla^{\mu} T_{\mu \nu}=$ 0 . Therefore for a diffeomorphism invariant action, $T_{\mu \nu}$ is conserved by virtue of the dynamic field equations and (32) may be used to define the stress-energy tensor of the field $\psi$. The above reasoning applied to $S_{G}$ gives $\nabla^{\mu} G_{\mu \nu}=0$ independently on any field equations, so that the contracted Bianchi identities follow from the invariance of the Hilbert action under diffeomorphisms [50].

The stress-energy tensor given by (32) is the metric stress-energy tensor for the case when $\mathscr{L}_{M}$ depends only on metric tensor $g_{\mu \nu}$. In general case it can depend also on its derivatives $g_{\mu \nu, \beta}$, and in this case it differs from the canonical stress-energy tensor introduced by variation of $\mathscr{L}_{M}$ with respect to dynamical variables describing the matter field. For electromagnetic field the Lagrangian $\mathscr{L}(F)$ does not depend on the derivatives of the metric tensor and can be reduced to the metric stress-energy tensor (32) by symmetrization $[49,50]$.

\section{Conflict of Interests}

The authors declare that there is no conflict of interests regarding the publication of this paper.

\section{References}

[1] H. A. Lorentz, Theory of Electrons, Dover, New York, NY, USA, 1952.

[2] P. A. M. Dirac, "An extensible model of the electron," Proceedings of the Royal Society of London A, vol. 268, pp. 57-67, 1962.

[3] E. T. Newman, E. Couch, K. Chinnapared, A. Exton, A. Prakash, and R. Torrence, "Metric of a rotating, charged mass," Journal of Mathematical Physics, vol. 6, pp. 918-919, 1965.

[4] B. Carter, "Global structure of the Kerr family of gravitational fields," Physical Review, vol. 174, no. 5, pp. 1559-1571, 1968. 
[5] C. A. López, "Extended model of the electron in general relativity," Physical Review D, vol. 30, no. 3, pp. 313-316, 1984.

[6] W. Israel, "Source of the Kerr metric," Physical Review D: Particles and Fields, vol. 2, pp. 641-646, 1970.

[7] A. Y. Burinskii, "Microgeons with spin," Soviet Physics-JETP, vol. 39, p. 193, 1974.

[8] V. H. Hamuty, "An 'interior' of the Kerr metric," Physics Letters A, vol. 56, no. 2, pp. 77-78, 1976.

[9] C. A. López, "Material and electromagnetic sources of the KerrNewman geometry," II Nuovo Cimento B Series 11, vol. 76, no. 1, pp. 9-27, 1983.

[10] V. de la Cruz, J. E. Chase, and W. Israel, "Gravitational collapse with asymmetries," Physical Review Letters, vol. 24, no. 8, pp. 423-426, 1970.

[11] J. M. Cohen, "Note on the Kerr Metric and Rotating Masses," Journal of Mathematical Physics, vol. 8, p. 1477, 1967.

[12] T. H. Boyer, "Quantum electromagnetic zero-point energy of a conducting spherical shell and the casimir model for a charged particle," Physical Review, vol. 174, no. 5, pp. 1764-1776, 1968.

[13] R. H. Boyer, "Rotating fluid masses in general relativity," Mathematical Proceedings of the Cambridge Philosophical Society, vol. 61, no. 2, pp. 527-530, 1965.

[14] R. H. Boyer, "Rotating fluid masses in general relativity. II," Proceedings of the Cambridge Philosophical Society, vol. 62, no. 3, pp. 495-501, 1966.

[15] M. Trümper, "Einsteinsche Feldgleichungen für das axialsymmetrische stationäre Gravitiationsfeld im Innern einer starr rotierenden idealen Flüssigkeit," Zeitschrift für Naturforschung, vol. 22, p. 1347, 1967.

[16] J. Tiomno, "Electromagnetic field of rotating charged bodies," Physical Review D, vol. 7, no. 4, pp. 992-997, 1973.

[17] A. Y. Burinskii,, "The problem of the source of the KerrNewman metric: the volume Casimir effect and superdense pseudovacuum state," Physics Letters B, vol. 216, no. 1-2, pp. 123126, 1989.

[18] A. Burinskii, "Supersymmetric superconducting bag as a core of Kerr spinning particle," Gravitation and Cosmology, vol. 8, pp. 261-271, 2002.

[19] A. Burinskii, E. Elizalde, S. R. Hildebrandt, and G. Magli, "Regular sources of the Kerr-Schild class for rotating and nonrotating black hole solutions," Physical Review D, vol. 65, no. 6, Article ID 064039, 2002.

[20] A. Burinskii, "Regularized Kerr-Newman solution as a gravitating soliton," Journal of Physics A: Mathematical and Theoretical, vol. 43, no. 39, Article ID 392001, 2010.

[21] A. Burinskii, "Stringlike structures in Kerr-Schild geometry: the $N=2$ string, twistors, and the Calabi-Yau twofold," Theoretical and Mathematical Physics, vol. 177, pp. 1492-1504, 2013.

[22] A. Burinskii, "Gravitational strings beyond quantum theory: electron as a closed heterotic string," Journal of Physics: Conference Series, vol. 361, Article ID 012032, 2012.

[23] J. D. Brown and J. W. York, Mathematical Aspects of Classical Field Theory, edited by M. J. Gotay, J. E. Marsden and V. Moncrief, American Mathematical Society, Providence, RI, USA, 1992.

[24] J. D. Brown and J. W. York, "Quasilocal energy and conserved charges derived from the gravitational action," Physical Review D, vol. 47, no. 4, pp. 1407-1419, 1993.

[25] E. S. Fradkin and A. A. Tseytlin, "Nonlinear electrodynamics from quantized strings," Physics Letters B, vol. 163, no. 1-4, pp. 123-130, 1985.
[26] A. A. Tseytlin, "Vector field effective action in the open superstring theory," Nuclear Physics B, vol. 276, no. 2, pp. 391-428, 1986.

[27] N. Seiberg and E. Witten, "String theory and noncommutative geometry," Journal of High Energy Physics, vol. 1999, article 032, 1999.

[28] M. Born and L. Infeld, "Foundation of the new field theory," Proceedings of the Royal Society of London A, vol. 144, pp. 425451, 1934

[29] I. Dymnikova, "Spinning superconducting electrovacuum soliton," Physics Letters B, vol. 639, no. 3-4, pp. 368-372, 2006.

[30] K. A. Bronnikov, "Regular magnetic black holes and monopoles from nonlinear electrodynamics," Physical Review D, vol. 63, no. 4, Article ID 044005, 6 pages, 2001.

[31] S. W. Hawking and G. F. R. Ellis, The Large Scale Structure of Space-Time, Cambridge University Press, London, UK, 1973.

[32] I. G. Dymnikova, "Vacuum nonsingular black hole," General Relativity and Gravitation, vol. 24, no. 3, pp. 235-242, 1992.

[33] I. G. Dymnikova, "The algebraic structure of a cosmological term in spherically symmetric solutions," Physics Letters. B, vol. 472, no. 1-2, pp. 33-38, 2000.

[34] I. Dymnikova, "The cosmological term as a source of mass," Classical and Quantum Gravity, vol. 19, no. 4, pp. 725-739, 2002.

[35] I. Dymnikova, "Spherically symmetric space-time with regular de Sitter center," International Journal of Modern Physics. D. Gravitation, Astrophysics, Cosmology, vol. 12, no. 6, pp. 10151034, 2003.

[36] I. Dymnikova, "Regular electrically charged vacuum structures with de Sitter centre in nonlinear electrodynamics coupled to general relativity," Classical and Quantum Gravity, vol. 21, no. 18, pp. 4417-4428, 2004.

[37] Ø. Grøn, "Repulsive gravitation and electron models," Physical Review D, vol. 31, no. 8, pp. 2129-2131, 1985.

[38] I. Dymnikova, A. Sakharov, and J. Ulbricht, "Appearance of a minimal length in $\mathrm{e}^{+} \mathrm{e}^{-}$annihilation," Advances in High Energy Physics, vol. 2014, Article ID 707812, 9 pages, 2014.

[39] R. P. Kerr and A. Schild, "Some algebraically degenerate solutions of Einstein's gravitational field equations," Proceedings of Symposia in Applied Mathematics, vol. 17, article 199, 1965.

[40] E. Elizalde and S. R. Hildebrandt, "Family of regular interiors for nonrotating black holes with $T_{0}^{0}=T_{1}^{1}$, Physical Review $D$, vol. 65, Article ID 124024, 2002.

[41] M. Gürses and F. Gürsey, "Lorentz covariant treatment of the Kerr-Schild geometry," Journal of Mathematical Physics, vol. 16, no. 12, pp. 2385-2390, 1975.

[42] D. V. Ahluwalia-Khalilova and I. Dymnikova, "A theoretical case for negative mass-square for sub-eV particles," International Journal of Modern Physics D, vol. 12, no. 9, pp. 1787-1794, 2003.

[43] I. Dymnikova, "Spacetime symmetry and mass of a lepton," Journal of Physics A: Mathematical and Theoretical, vol. 41, no. 30, Article ID 304033, 2008.

[44] E. Ayón-Beato and A. García, "Regular black hole in general relativity coupled to nonlinear electrodynamics," Physical Review Letters, vol. 80, no. 23, pp. 5056-5059, 1998.

[45] E. Ayón-Beato and A. García, "New regular black hole solution from nonlinear electrodynamics," Physics Letters B, vol. 464, no. 1-2, pp. 25-29, 1999.

[46] E. Ayon-Beato and A. Garcia, "Non-singular charged black hole solution for non-linear source," General Relativity and Gravitation, vol. 31, no. 5, pp. 629-633, 1999. 
[47] E. Ayon-Beato and A. Garcia, "Four-parametric regular black hole solution," General Relativity and Gravitation, vol. 37, no. 4, pp. 635-641, 2005.

[48] H. Salazar, A. Garcia, and J. Plebański, "Duality rotations and type D solutions to Einstein equations with nonlinear electromagnetic sources," Journal of Mathematical Physics, vol. 28, no. 9, pp. 2171-2181, 1987.

[49] L. D. Landau and E. M. Lifshitz, Classical Theory of Fields, Pergamon Press, 1975.

[50] R. M. Wald, General Relativity, University of Chicago Press, 1984.

[51] K. Bronnikov, I. Dymnikova, and E. Galaktionov, "Multihorizon spherically symmetric spacetimes with several scales of vacuum energy," Classical and Quantum Gravity, vol. 29, no. 9, Article ID 095025, 2012.

[52] I. Dymnikova, E. Galaktionov, and E. Tropp, "Lagrangians in nonlinear electrodynamics minimally coupled to gravity".

[53] I. G. Dymnikova, "De Sitter-SCHwarzschild black hole: its particlelike core and thermodynamical properties," International Journal of Modern Physics D, vol. 5, no. 5, pp. 529-540, 1996.

[54] I. Dymnikova and M. Korpusik, "Regular black hole remnants in de Sitter space," Physics Letters B, vol. 685, no. 1, pp. 12-18, 2010.

[55] A. Burinskii and S. R. Hildebrandt, "New type of regular black holes and particlelike solutions from nonlinear electrodynamics," Physical Review D, vol. 65, no. 10, Article ID 104017, 2002. 


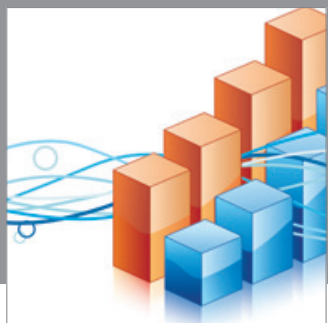

Advances in

Operations Research

mansans

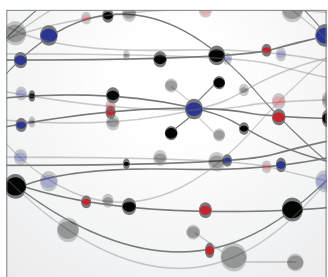

The Scientific World Journal
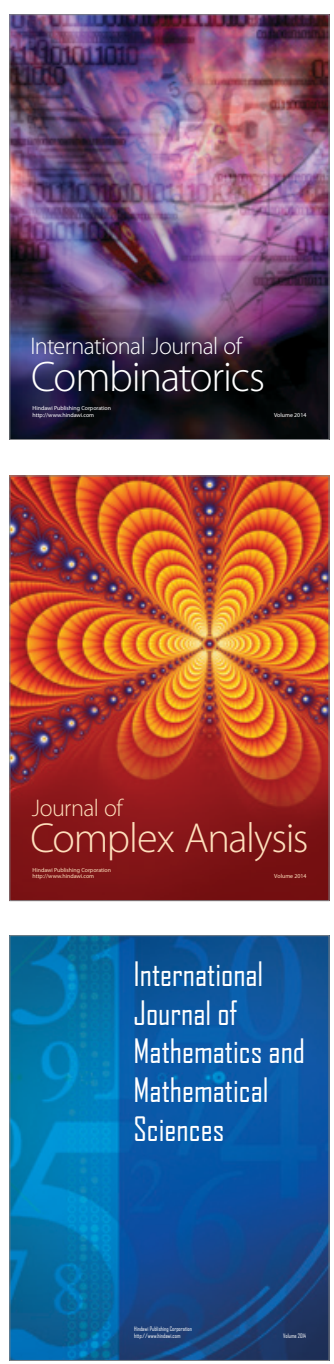
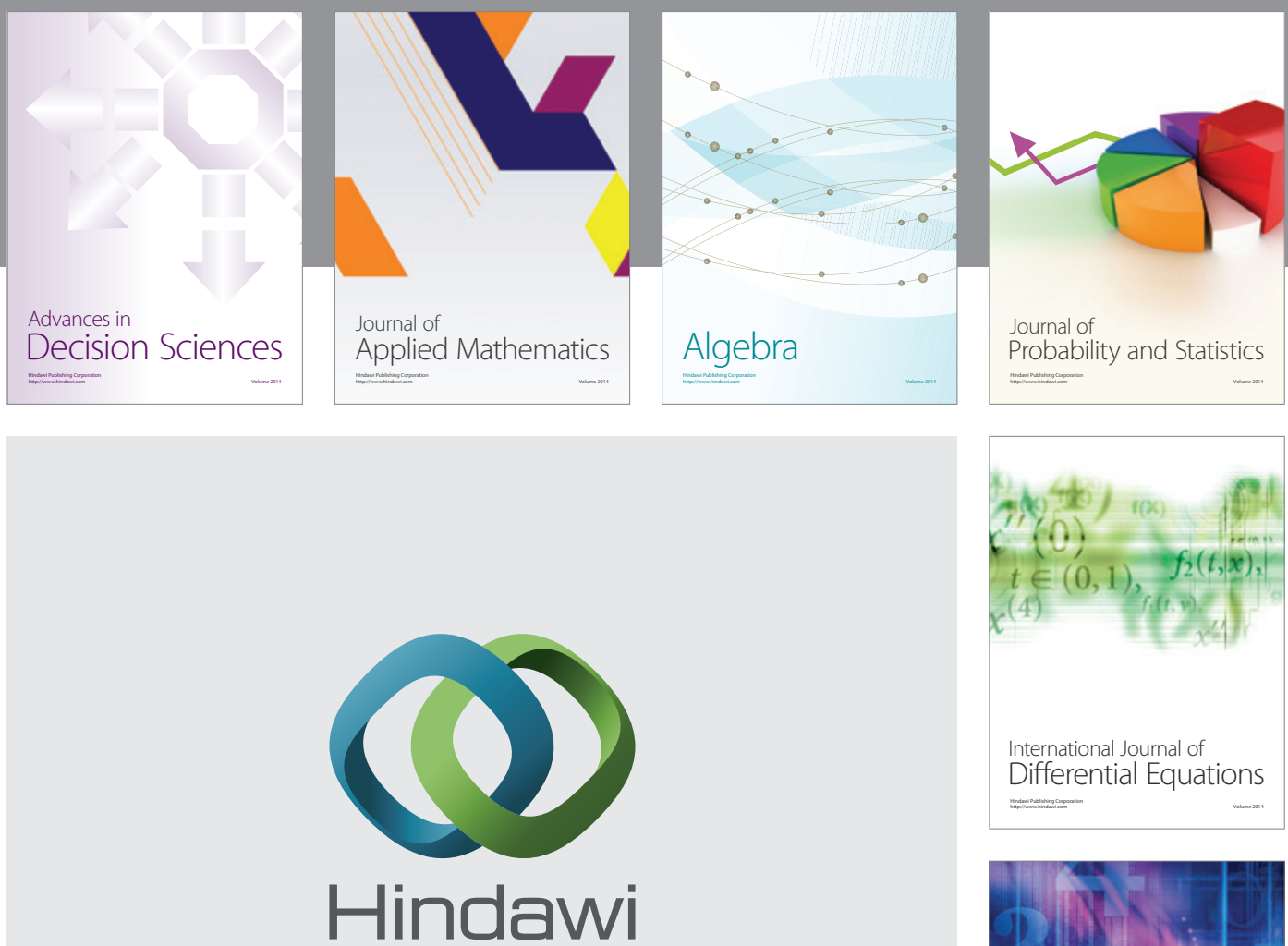

Submit your manuscripts at http://www.hindawi.com
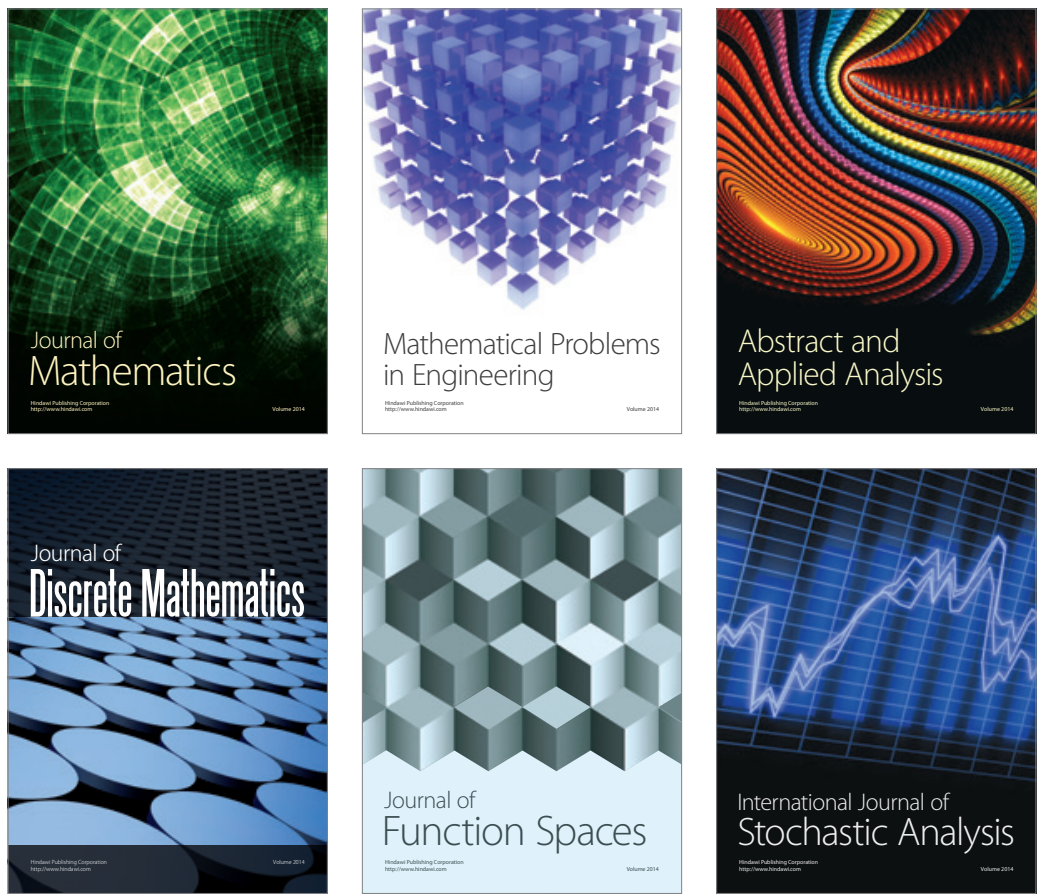

Journal of

Function Spaces

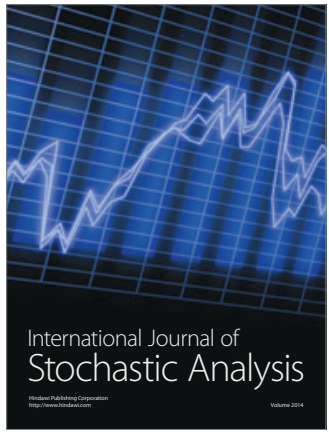

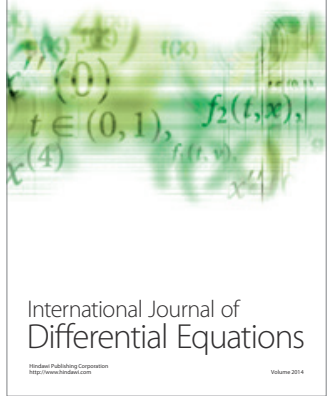
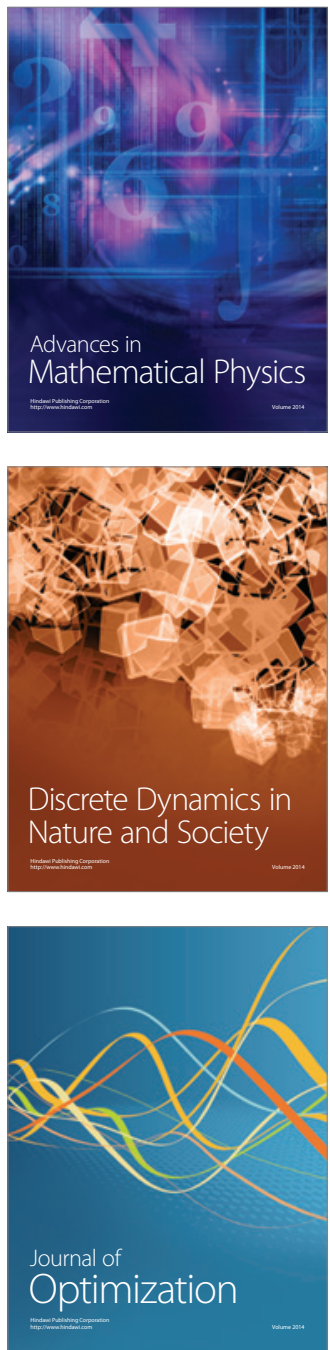